\title{
Alpha-defensins determination in different types of synovial fluid and parallel collected serum samples by ELISA
}

\author{
Pavlina Kusnierovaa ${ }^{\mathrm{a}}$, Iveta Bystronovaa ${ }^{\mathrm{a}, \mathrm{b}}$, Pavel Walder', Rudolf Hlubekc, Frantisek Vsiansky ${ }^{\mathrm{a}}$, David Stejskal ${ }^{\mathrm{a}}$
}

\begin{abstract}
Aims. The study aims were to verify the serum (S) and synovial fluid (SF) reference intervals (RIs) for human neutrophil defensins (HNP1-3); measure S and SF defensin concentrations in different types of SF, including non-inflammatory, inflammatory non-pyogenic, inflammatory pyogenic, and hemorrhagic; and to compare the HNP1-3 concentrations in SF and S with those of other inflammatory biomarkers.

Methods. SF and S samples were collected from 92 patients. HNP1-3 concentrations were determined using enzymelinked immunosorbent assays; glucose, lactate, interleukin-6, and procalcitonin using an automatic analyzer; and presepsin using a Pathfast system. There were 61 non-inflammatory, 11 inflammatory non-pyogenic, 11 inflammatory pyogenic, and 9 hemorrhagic SF. Non-inflammatory SF was divided into non-inflammatory normal and non-inflammatory osteoarthritis. The former was used to estimate the HNP1-3 RI in SF and S.

Results. The estimated HNP1-3 RIs of SF and S were 12.47-437.42 mg/L and 5.45-44.75 $\mu \mathrm{g} / \mathrm{L}$, respectively. HNP1-3 differed significantly between $S$ and SF and individual groups of $S F(P<0.001$ and $P=0.001$, respectively). There were significant relationships between SF HNP1-3 and S HNP1-3 $(P<0.001)$, S C-reactive protein $(P<0.001)$, and S interleukin-6 $(P=0.007)$, and between SF HNP1-3 and SF C-reactive protein $(P=0.004)$ and SF interleukin-6 $(P<0.001)$. The highest kappa coefficient was between SF HNP1-3 and SF interleukin-6 $(K=0.507)$.

Conclusions. We validated the SF HNP1-3 diagnostic kit and demonstrated that SF and S HNP1-3 are promising biomarkers for distinguishing inflammatory from non-inflammatory joint diseases.
\end{abstract}

Key words: alpha-defensins, synovial fluid, inflammatory joint disease, enzyme-linked immunosorbent assay

Received: June 10, 2020; Revised: April 6, 2021; Accepted: April 7, 2021; Available online: April 19, 2021

https://doi.org/10.5507/bp.2021.020

(c) 2021 The Authors; https://creativecommons.org/licenses/by/4.0/

aInstitute of Laboratory Diagnostics, Department of Clinical Biochemistry, University Hospital Ostrava, Czech Republic

${ }^{b}$ Department of Epidemiology and Public Health, Faculty of Medicine, University of Ostrava, Czech Republic

'Orthopedic Department, University Hospital Ostrava, Czech Republic

Corresponding author: Pavlina Kusnierova, e-mail: pavlina.kusnierova@fno.cz

\section{INTRODUCTION}

Joint effusion can have different etiologies, associated appearances, and biochemical and microscopic characteristics. Dungl et al. ${ }^{1}$ divides joint effusions into noninflammatory, inflammatory, and pyogenic. It is debatable whether any effusion (increase in intra-articular fluid) can be fully non-inflammatory. It is crucial to understand the nature of the process. It can be inflammation provoked by mechanical factors leading to cartilage abrasion and the production of microscopic cartilage particles (initially only as a manifestation of delamination of the surface layer of the cartilage). Inflammation can also occur due to chemicals, such as uric acid salts in hyperuricemic syndrome. Many systemic diseases also lead to arthritis with overproduction of joint fluid. However, the crucial fact is that the aforementioned types of inflammation are free of microorganisms.

Inflammatory effusions with the presence of live pathogens must be fundamentally distinguished from non-inflammatory exudates and require specific treatment procedures, often surgical and urgent. Modern diagnostic methods (including experimental) are focused on the diagnosis of these types of effusions. A common diagnostic method is the collection of the fluid for culture or prolonged culture and measurement of the difference in glucose level between the fluid and serum, or a similar difference in the levels of C-reactive protein and other markers. Diagnostics are performed using modern methods such as bacterial DNA detection using PCR techniques or the determination of new biochemical markers. Identification of the agent by cultivation is sometimes problematic, as the examination is often performed after untargeted antibiotic therapy has been used already. Diagnosis of the so-called mitigated infection is also problematic. Errors also occur during the collection and transport of media with a puncture, etc.

Many septic inflammations are accompanied by a general alteration of the host with a threat not only to the patient's health but also to their life. The pathogen cannot be expected to be detected and identified but, despite this, early surgical therapy should be indicated.

Rapid differentiation between septic and aseptic etiology can also be crucial in joint replacement effusions. Here, too, there are factors complicating the detection of pathogens: preventive administration of antibiotics 
(ATB), ATB in bone cement, untargeted ATB therapy, pathogens with low virulence, a good immune response to bacteria in free fluid that is insufficiently effective against bacteria in biofilm, etc. In the revision, it is then necessary to decide between septic and aseptic etiology, although the pathogen is often not proven.

In addition to the biomarkers mentioned above, current diagnostics are based on imaging methods such as computed tomography (CT or CAT), magnetic resonance imaging, and positron emission tomography, or state-ofthe-art combinations of methods such as SPECT/CT and PET/CT. However, most of these tests do not show 100\% sensitivity and specificity, so a combination of clinical history and symptoms is required. One of the biomarker that can be used is alpha-defensin.

Human neutrophil defensins (alpha-defensins, HNP13 ) belong to the family of low molecular weight (14-60 $\mathrm{kD}$ ) cationic peptides. They are localized on azurophilic granules and represent about $99 \%$ of the total defensin content in these cells. In addition to microbicidal effects, they exhibit chemotactic, immunomodulatory, and cytotoxic activity and are involved in host defense and inflammation. Human defensins are divided into alpha- and beta-defensins according to their structure. Alpha-defensins are found in granulocytes in the mucous membranes of the urogenital and intestinal tracts. Beta-defensins are present in all epithelial tissues. In the airway epithelium, beta-defensin 1 is produced constitutively and beta-defensins 2 and 3 are induced in response to infectious agents ${ }^{2-4}$. Neutrophil activation leads to the rapid release of defensins into both synovial fluid (SF) and plasma. Under physiological conditions, plasma or serum HNP1-3 concentrations should range from undetectable levels to about 50-100 $\mu \mathrm{g} / \mathrm{L}$. However, in septic conditions, plasma or serum levels increase dramatically and can reach values of up to $10 \mathrm{~g} / \mathrm{L}$ or more ${ }^{5}$.

Therefore, the aim of our study was to verify the reference intervals (RIs) of serum (S) and synovial fluid (SF) human neutrophil defensins and to measure their serum and synovial fluid concentrations in different groups including non-inflammatory, inflammatory, and hemorrhagic and to compare the concentrations of HNP1-3 in $\mathrm{SF}$ and $\mathrm{S}$ with those of other inflammatory biomarkers.

\section{MATERIALS AND METHODS}

\section{Patients}

Our study included 92 patients from the Orthopedic Department whose ST and S samples were sent for analysis to the Institute of Laboratory Diagnostics, Department of Clinical Biochemistry, University Hospital Ostrava. All patients provided signed informed consent prior to their involvement in the study. Approval was obtained by the Ethics Committee of the University Hospital Ostrava, the Czech Republic (reference number of project 322/2018), in accordance with the Helsinki Declaration of 1975 as revised in 2000. Patients were divided into individual groups according to Dungl et al. ${ }^{\text {: }}$ : non-inflammatory $S F(n=61$; average age $62.4 \pm 17.1$ years), inflammatory non-pyogenic (due to the emphasis on the absence of a pyogenic form of inflammation; $n=11$; average age 55.2 \pm 12.5 years $)$, inflammatory pyogenic $(n=11$; average age $61.6 \pm 10.8$ years), and hemorrhagic SF ( $n=9$; average age $58.2 \pm 14.7$ years). The non-inflammatory SF group was further divided into non-inflammatory normal SF $(n=31$; average age $59.8 \pm 15.3$ years) and non-inflammatory osteoarthritis $(n=30$; average age $65.1 \pm 18.7$ years $)$. Samples from the non-inflammatory normal SF group ( $n=31$, number of outliers $2 ; n=29$ ) were used to estimate the RI of SF HNP1-3 and of S HNP1-3.

\section{Samples}

SF samples were collected into polypropylene tubes (Sarstedt, Nümbrecht, Germany), and serum samples into Serum Gel with Clotting Activator tubes (Sarstedt). The synovial fluid and the serum were collected on the same day. A portion of the uncentrifuged synovial fluid sample was used for cytological analysis. The remainder of the synovial fluid and whole serum samples was centrifuged at $2500 \times \mathrm{g}$ for $6 \mathrm{~min}$ at $4{ }^{\circ} \mathrm{C}$, and the supernatants were then aliquoted into at least $3-5$ vials $(0.5 \mathrm{~mL}$ per vial $)$ and stored at $-70{ }^{\circ} \mathrm{C}$ until analyzed.

\section{Analytical methods}

The concentrations of HNP1-3 (Human HNP1-3 ELISA Kit, Ref HK317, Hycult Biotech, Inc. United States) were determined using ELISA. The concentrations of glucose (GLU, REF OSR6521, Beckman Coulter, Brea, CA, USA), lactate (LAC, REF OSR6193, Beckman Coulter), total protein (TP, REF OSR6232, Beckman Coulter) and C-reactive protein (CRP, REF OSR6147, Beckman Coulter) were determined using an AU 5800 automated analyzer. Hematological parameters, including relative and absolute numbers of leukocytes (WBC), erythrocytes (RBC), and mononuclear (MNP) and polymorphonuclear (PMN) leukocytes were counted using a Sysmex XN-9000 in body fluid mode. Interleukin 6 (IL-6, ADVIA Centaur IL-6, REF 10995080, Siemens) and procalcitonin (PCT, ADVIA Centaur BRAHMS Procalcitonin, REF 10378883, Siemens) were determined using an ADVIA Centaur XP automated analyzer, and presepsin (PRES, Pathfast Presepsin, REF 1110-4000, Mitsubishi Kagaku Iatron, Inc.) on a Pathfast system.

Diluent solution and a patient's SF and S samples at very low concentration were used to estimate the blank limit (LoB) and the detection limit (LoD). Diluted standard samples were used for precise and reproducible measurement of HNP1-3, because the diagnostic kit did not include a quality control sample. To verify linearity, SF and $\mathrm{S}$ samples of low (L) and high concentration $(\mathrm{H})$ were used, and were subsequently diluted in appropriate proportions.

Undiluted SF was used for the determination of routine and inflammatory biomarkers on the automated analyzer. For S HNP1-3 and SF HNP1-3, samples were used at $1 / 20$ and $1 / 1000$ dilution, respectively.

Kit manufacturers reported that the detection level of HNP1-3 was <156 ng/L and recovery between $85.5 \%$ and $91.5 \%$. 


\section{Statistical methods}

The software packages Excel, Stata version 13, MedCal version 17.9.7, R, and NCSS 2007 were used for statistical analysis ${ }^{6-7}$. To estimate the HNP1-3 reference interval in synovial fluid and serum, both MedCal version 17.9.7 and the robust CLSI C28-A3 method were used, due to the small sample sizes $(n=29$ and $n=25$ for the $S F$ and S HNP1-3 reference ranges, respectively).

Basic descriptive statistics, including frequency tables, medians, arithmetic means, standard deviations and percentiles, were used to describe the results. The normality criteria were rejected; therefore, non-parametric tests were used, including the Kruskal-Wallis rank test followed by Dunn's multiple pairwise comparisons to identify which groups were different with Bonferroni p-adjusted correction.

The relationship between the parameters was assessed using Spearman's correlation coefficient. Conformity between assay results was measured using the kappa index with confidence intervals of $95 \%$. Statistical tests were evaluated using a significance level of $5 \%$.

RIs were estimated based on the guidelines of the Clinical and Laboratory Standards Institute (CLSI C28-A3), which recommend the use of nonparametric tests for statistical data processing ${ }^{8}$.

\section{RESULTS}

Information regarding the validation of the diagnostic kit for the determination of HNP1-3 is shown in Table 1. Based on repeated measurements $(n=10)$ of the blank and the low-concentration HNP1-3 sample, the limit of blank $(\mathrm{LoB}=82.66 \mu \mathrm{g} / \mathrm{L})$ and the limit of detection $(\mathrm{LoD}=109.37 \mu \mathrm{g} / \mathrm{L})$ values were calculated. The average recovery of HNP1-3 obtained using spiked samples of SF was $99.4 \%$.

Table 1. Assessment of the precision of a commercial HNP1-3 ELISA kit using patient synovial fluid samples.

\begin{tabular}{lcccc}
\hline & \multicolumn{2}{c}{ Intra-Assay Precision } & \multicolumn{2}{c}{ Inter-Assay Precision } \\
Control & Level 1 & Level 2 & Level 1 & Level 2 \\
\hline $\mathrm{n}$ & 10 & 10 & 5 & 5 \\
Mean $(\mathrm{mg} / \mathrm{L})$ & 517 & 2283 & 683 & 10342 \\
$\mathrm{SD}(\mathrm{mg} / \mathrm{L})$ & 17.38 & 95.79 & 18.58 & 595.47 \\
$\mathrm{CV}(\%)$ & 3.36 & 4.20 & 2.72 & 5.76 \\
\hline
\end{tabular}

$\mathrm{n}$, number of patients; SD, standard deviation; CV (\%), coefficient of variation

Table 2. Descriptive characteristics; values of means \pm standard deviation estimated in individual groups of patients.

\begin{tabular}{|c|c|c|c|c|c|c|c|c|}
\hline & \multicolumn{8}{|c|}{ Diagnostic groups } \\
\hline & \multicolumn{2}{|c|}{ Non-Inflammatory } & \multicolumn{2}{|c|}{$\begin{array}{l}\text { Inflammatory- } \\
\text { non-pyogenic }\end{array}$} & \multicolumn{2}{|c|}{$\begin{array}{l}\text { Inflammatory- } \\
\text { pyogenic }\end{array}$} & \multicolumn{2}{|c|}{ Hemorrhagic } \\
\hline & $\mathrm{n}$ & Mean \pm SD & $\mathrm{n}$ & Mean \pm SD & $\mathrm{n}$ & Mean \pm SD & $\mathrm{n}$ & Mean \pm SD \\
\hline Age (years) & 61 & $62.4 \pm 17.1$ & 11 & $55.2 \pm 12.5$ & 11 & $61.6 \pm 10.8$ & 9 & $58.2 \pm 14.7$ \\
\hline SF GLU (mmol/L) & 58 & $4.81 \pm 2.24$ & 10 & $4.40 \pm 2.35$ & 10 & $3.51 \pm 2.42$ & 8 & $4.90 \pm 1.43$ \\
\hline SF LAC (mmol/L) & 59 & $2.99 \pm 1.76$ & 10 & $3.93 \pm 2.21$ & 10 & $8.19 \pm 3.13$ & 8 & $4.02 \pm 1.93$ \\
\hline SF KEB (-) & 57 & $-4.91 \pm 220$ & 10 & $-45.7 \pm 182$ & 10 & $-666 \pm 2057$ & 8 & $17.9 \pm 23.5$ \\
\hline ST TP $(\mathrm{g} / \mathrm{L})$ & 45 & $34.9 \pm 11.0$ & 9 & $41.8 \pm 10.6$ & 6 & $42.8 \pm 11.7$ & 8 & $40.6 \pm 8.94$ \\
\hline ST CRP (mg/L) & 60 & $2.19 \pm 2.94$ & 10 & $18.5 \pm 28.5$ & 11 & $34.3 \pm 29.9$ & 8 & $21.0 \pm 21.6$ \\
\hline ST IL-6 $(\mu \mathrm{g} / \mathrm{L})$ & 39 & $1.41 \pm 3.40$ & 4 & $0.21 \pm 0.38$ & 9 & $49.6 \pm 16.1$ & 9 & $18.2 \pm 24.3$ \\
\hline ST PCT $(\mu \mathrm{g} / \mathrm{L})$ & 35 & $0.60 \pm 0.09$ & 3 & $0.73 \pm 0.06$ & 9 & $0.72 \pm 0.33$ & 8 & $0.58 \pm 0.14$ \\
\hline ST PRES $(\mu \mathrm{g} / \mathrm{L})$ & 59 & $3.72 \pm 3.79$ & 10 & $5.98 \pm 7.34$ & 10 & $3.61 \pm 3.84$ & 7 & $2.57 \pm 2.40$ \\
\hline ST HNP1-3 (mg/L) & 61 & $635 \pm 1963$ & 11 & $1691 \pm 1806$ & 11 & $6129 \pm 2696$ & 9 & $4926 \pm 6287$ \\
\hline $\mathrm{SF} \mathrm{WBC} * 10^{6} / \mathrm{L}$ & 60 & $635 \pm 1126$ & 11 & $12420 \pm 10198$ & 10 & $33668 \pm 21915$ & 9 & $8293 \pm 13899$ \\
\hline PMN (\%) & 60 & $27.4 \pm 16.2$ & 11 & $58.5 \pm 26.9$ & 10 & $87.8 \pm 13.2$ & 9 & $62.8 \pm 29.3$ \\
\hline $\mathrm{S}$ GLU (mmol/L) & 44 & $5.91 \pm 1.60$ & 6 & $6.25 \pm 1.55$ & 9 & $6.65 \pm 4.23$ & 7 & $5.70 \pm 0.90$ \\
\hline P LAC (mmol/L) & 24 & $1.56 \pm 0.50$ & 2 & $0.87 \pm 0.21$ & 5 & $2.04 \pm 0.69$ & 7 & $1.68 \pm 0.44$ \\
\hline $\mathrm{S}$ CRP (mg/L) & 44 & $4.58 \pm 4.18$ & 6 & $14.8 \pm 20.4$ & 9 & $75.3 \pm 37.9$ & 7 & $56.5 \pm 54.1$ \\
\hline S IL-6 (ng/L) & 34 & $17.6 \pm 21.2$ & 3 & $14.3 \pm 2.52$ & 9 & $50.1 \pm 74.5$ & 7 & $147 \pm 227$ \\
\hline S PCT $(\mu \mathrm{g} / \mathrm{L})$ & 34 & $0.04 \pm 0.1352$ & 3 & $0.01 \pm 0.00$ & 9 & $0.11 \pm 0.22$ & 7 & $0.32 \pm 0.61$ \\
\hline S PRES (ng/L) & 40 & $2001 \pm 178$ & 6 & $741 \pm 1506$ & 8 & $304 \pm 238$ & 6 & $243 \pm 77.7$ \\
\hline S HNP1-3 (mg/L) & 44 & $13.8 \pm 7.68$ & 6 & $15.6 \pm 10.4$ & 9 & $24.9 \pm 4.63$ & 7 & $20.5 \pm 6.96$ \\
\hline
\end{tabular}

GLU, glucose; LAC, lactate; KEB, coefficient of energetic balance; CRP, C-reactive protein; IL-6, interleukin 6; PCT, procalcitonin; PRES, presepsin; HNP1-3, human neutrophil defensins 1-3; WBC, leukocytes; PMN, polymorphonuclear; P, plasma; P, plasma; n, number of patients; $\mathrm{SD}$; standard deviation 
The estimated RI of SF HNP1-3 was 12.47-437.42 $\mathrm{mg} / \mathrm{L}$ and of S HNP1-3 was 5.45-44.75 $\mu \mathrm{g} / \mathrm{L}$ (Fig. 1).

A total of 92 patient samples were included in the analysis, which evaluated the correlation between HNP1-3 levels and several other biochemical markers. Characteristics of the studied groups are presented in Table 2.

A statistically significant difference between SF HNP1-3 and the individual SF type groups $(P<0.0001)$ and between S HNP1-3 and the individual groups of SF type $(P=0.0006)$ was found by use of the nonparametric Kruskal-Wallis test (Fig. 2).

The regression relationship between studied parameters was evaluated using Passing-Bablok regression (Fig. 3). There was a statistically significant correlation between SF HNP1-3 and S HNP1-3 concentrations ( $\mathrm{r}_{\mathrm{s}}=0.493, \mathrm{n}=66, P<0.001$ ), between SF HNP1-3 and $\mathrm{S}$ CRP concentrations $\left(\mathrm{r}_{\mathrm{s}}=0.609, \mathrm{n}=66, P<0.001\right)$, between SF HNP $1-3$ and S IL-6 concentrations $\left(r_{s}=0.366, n=53\right.$, $P=0.007)$, between SF HNP1-3 and S PCT concentrations $\left(\mathrm{r}_{\mathrm{s}}=0.375, \mathrm{n}=53, P=0.006\right)$, between SF HNP1-3 and SF CRP concentrations $\left(\mathrm{r}_{\mathrm{s}=} 0.304, \mathrm{n}=89, P=0.004\right)$, and between SF HNP1-3 and SF IL-6 concentrations $\left(\mathrm{r}_{\mathrm{s}=} 0.558, \mathrm{n}=57, P<0.001\right)$ (Table 3$)$.

Cohen's kappa statistic was used to test interrater reliability (Table 4). The highest kappa coefficient, indicating moderate conformity between the studied biomarkers, was between the concentrations of SF HNP1-3 and SF IL-6 $(\kappa=0.507)$. According to Cohen's interpretation for healthrelated studies, this score is acceptable ${ }^{6}$.

Table 3. Correlations between selected biochemical markers in SF and S and individual groups.

\begin{tabular}{|c|c|c|c|c|c|c|}
\hline \multirow[t]{2}{*}{ Parameters } & & \multicolumn{5}{|c|}{ Studies groups } \\
\hline & & Hemorrhagic & $\begin{array}{l}\text { Inflammatory } \\
\text {-non-pyogenic }\end{array}$ & $\begin{array}{l}\text { Inflammatory } \\
\text {-pyogenic }\end{array}$ & Non-Inflammatory & All \\
\hline \multirow{3}{*}{$\begin{array}{l}\text { SF HNP1-3 vs. } \\
\text { S HNP1-3 }\end{array}$} & $\mathrm{n}$ & 7 & 6 & 9 & 44 & 66 \\
\hline & $r_{s}$ & 0.536 & 0.486 & 0.783 & 0.136 & 0.493 \\
\hline & $\stackrel{\mathrm{s}}{P}$ & 0.215 & 0.329 & 0.013 & 0.380 & $<0.001$ \\
\hline \multirow{3}{*}{$\begin{array}{l}\text { SF HNP1-3 vs. } \\
\text { S CRP }\end{array}$} & $\mathrm{n}$ & 7 & 6 & 9 & 44 & 66 \\
\hline & $r_{s}$ & 0.714 & 0.600 & 0.536 & 0.087 & 0.609 \\
\hline & $P$ & 0.071 & 0.208 & 0.137 & 0.573 & $<0.001$ \\
\hline \multirow{3}{*}{$\begin{array}{l}\text { SF HNP1-3 vs. } \\
\text { S IL-6 }\end{array}$} & $\mathrm{n}$ & 7 & 3 & 9 & 34 & 53 \\
\hline & $\mathrm{r}_{\mathrm{s}}$ & 0.893 & 1.000 & 0.400 & 0.108 & 0.366 \\
\hline & $\stackrel{\mathrm{s}}{P}$ & 0.007 & - & 0.286 & 0.541 & 0.007 \\
\hline \multirow{3}{*}{$\begin{array}{l}\text { SF HNP1-3 vs. } \\
\text { S PCT }\end{array}$} & $\mathrm{n}$ & 7 & & 9 & 34 & 53 \\
\hline & $\mathrm{r}_{\mathrm{s}}$ & 0.579 & - & 0.253 & -0.197 & 0.375 \\
\hline & $\stackrel{s}{P}$ & 0.173 & & 0.511 & 0.264 & 0.006 \\
\hline \multirow{3}{*}{$\begin{array}{l}\text { SF HNP1-3 vs. } \\
\text { S PRES }\end{array}$} & $\mathrm{n}$ & 6 & 6 & 8 & 40 & 60 \\
\hline & $r_{s}$ & 0.714 & 0.754 & 0.690 & -0.229 & 0.184 \\
\hline & $\stackrel{\mathrm{s}}{P}$ & 0.111 & 0.084 & 0.058 & 0.155 & 0.158 \\
\hline \multirow{3}{*}{$\begin{array}{l}\text { SF HNP1-3 vs. } \\
\text { SF CRP }\end{array}$} & $\mathrm{n}$ & 8 & 10 & 11 & 60 & 89 \\
\hline & $r_{s}$ & 0.119 & -0.321 & 0.200 & -0.162 & 0.304 \\
\hline & $\stackrel{s}{P}$ & 0.779 & 0.366 & 0.555 & 0.215 & 0.004 \\
\hline \multirow{3}{*}{$\begin{array}{l}\text { SF HNP1-3 vs. } \\
\text { SF IL-6 }\end{array}$} & $\mathrm{n}$ & 8 & 3 & 9 & 39 & 57 \\
\hline & $\mathrm{r}_{\mathrm{s}}$ & 0.838 & 1.000 & 0.000 & -0.229 & 0.558 \\
\hline & $\stackrel{s}{P}$ & 0.009 & & 1.000 & 0.161 & $<0.001$ \\
\hline \multirow{3}{*}{$\begin{array}{l}\text { SF HNP 1-3 vs. } \\
\text { SF PCT }\end{array}$} & $\mathrm{n}$ & 8 & 3 & 9 & 35 & 55 \\
\hline & $r_{s}$ & -0.275 & -0.866 & 0.347 & -0.019 & -0.068 \\
\hline & $\stackrel{s}{P}$ & 0.510 & 0.333 & 0.360 & 0.914 & 0.622 \\
\hline \multirow{3}{*}{$\begin{array}{l}\text { SF HNP 1-3 vs. } \\
\text { SF PRES }\end{array}$} & $\mathrm{n}$ & 7 & 10 & 10 & 59 & 86 \\
\hline & $r_{s}$ & 0.107 & 0.636 & -0.418 & 0.294 & 0.153 \\
\hline & $\stackrel{s}{P}$ & 0.819 & 0.048 & 0.229 & 0.024 & 0.158 \\
\hline
\end{tabular}

$\mathrm{r}_{\mathrm{s},}$ Spearman's correlation coefficient; $P$, probability; $\mathrm{n}$, number of values

Table 4. Correlation of selected parameters based on positivity and negativity of results, Cohen's kappa statistics.

\begin{tabular}{lccccc}
\hline & SF HNP1-3 vs. & SF HNP1-3 vs. & SF HNP1-3 vs. & SF HNP1-3 vs. & SF HNP 1-3 vs. \\
& S HNP1-3 & S CRP & S IL-6 & SF CRP & SF IL-6 \\
\hline Weighted Kappa $(\mathrm{n})$ & 0.352 & 0.300 & 0.071 & 0.360 & 0.507 \\
& $(66)$ & $(87)$ & $(83)$ & $(92)$ & $(64)$ \\
95\% CI & $0.093-0.571$ & $0.155-0.446$ & $-0.049-0.190$ & $0.179-0.542$ & $0.292-0.722$ \\
Standard error & 0.122 & 0.074 & 0.061 & 0.093 & 0.110 \\
\hline
\end{tabular}

Positive values were as follows: SF HNP1-3 > 437.42 mg/L; S HNP1-3 44.75 mg/L; S CRP > 8.0 mg/L (ref. ${ }^{9}$ ); SF CRP* > 9.31 mg/L; S IL-6 > $4.4 \mathrm{ng} / \mathrm{L}\left(\right.$ ref. $^{10}$ ); SF IL-6* $>12 \mu \mathrm{g} / \mathrm{L}$ 

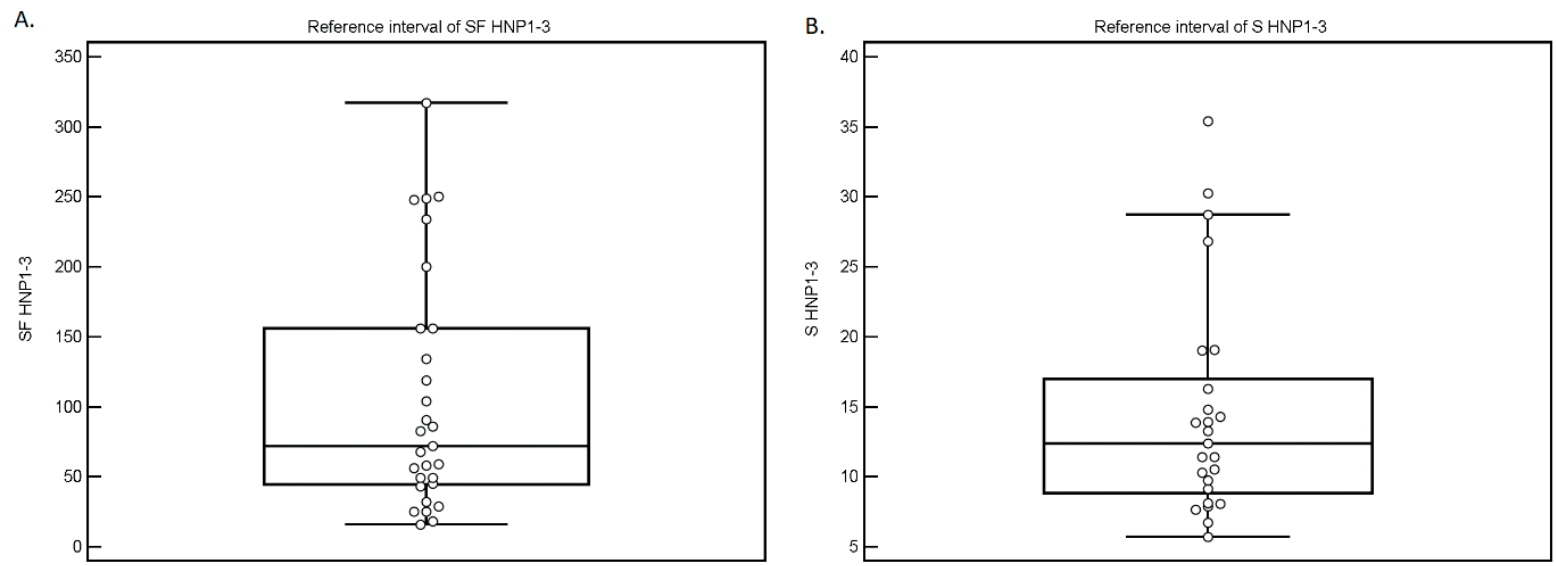

Fig. 1. Estimation of the SF HNP1-3 (A) and S HNP1-3 (B) reference intervals.
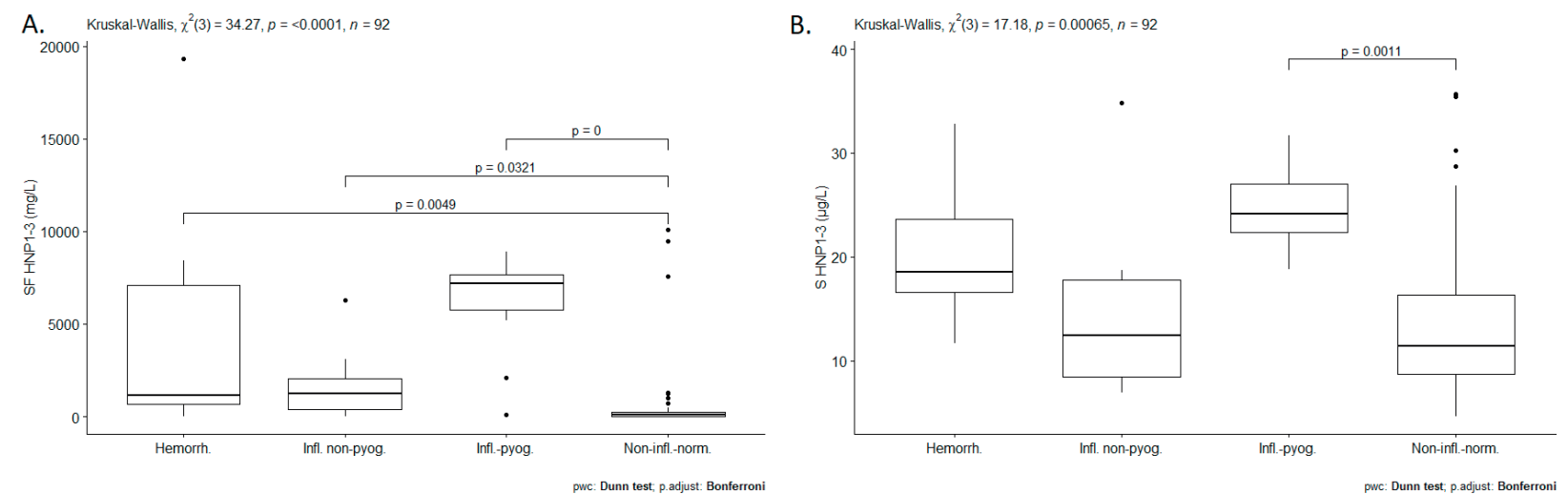

Fig. 2. Box-plot of ST HNP1-3 (A) and S HNP1-3 (B) in individual groups according to type of synovial fluid.

\section{DISCUSSION}

In this study, we tested HNP1-3 as a marker of inflammatory joint diseases. The HNP1-3 ELISA diagnostics kit is sensitive enough for measurement in serum and synovial fluid. The estimated reference limits for serum HNP1-3 roughly correspond to the values presented by Kemik et al. and Qi et al. ${ }^{11-12}$. These authors studied this marker, among others, in plasma in healthy volunteers. The mean plasma HNP1-3 levels were $68 \pm 22 \mathrm{ng} / \mathrm{mL}$ and approximately $50 \mathrm{ng} / \mathrm{mL}$, respectively. This partial difference may be due to the fact that our values were determined in serum, whereas Kemik et al. and Qi et al. used plasma. Subsequently, we estimated reference limits for SF HNP1-3 in the non-inflammatory normal SF group. Then we compared the SF HNP1-3 and S HNP1-3 concentrations between individual groups of the SF type, characterizing the joint effusions according to Dungl et al. ${ }^{1}$. To limit the possibility for errors, we called the inflammatory category "inflammatory non-pyogenic" (to emphasize the absence of the pyogenic form of inflammation). The classification into individual groups is, of course, possible according to the given parameters, but as already mentioned in the introduction, an error may occur, due either to failure to detect the microorganism or to contamination of the sample from the skin or ex vivo. In our experience, the highest degree of probability is given by a combination of results, especially over the time course of the disease. All our subjects were monitored for at least half a year after the tests, and the development of their disease allowed us to included them in individual groups with a probability close to certainty. For example, their categorization was confirmed with subsequent culture of pathogen in the pyogenic inflammation group, healing of exudates in the "non-inflammatory" group without ATB therapy but only the use of common procedures such as corticoid application, or the solution of mechanical or degenerative changes through surgical therapy (arthroscopically). For a group of inflammatory non-pyogenic effusions, anamnestic finding of another cause and repeated negative findings of the microorganism in the monitored effusion were confirmatory. In our study, statistically significant differences between these groups were demonstrated. A similar result was described by Melicherčík et al. ${ }^{13}$. These authors studied this marker in the synovial fluid of 157 patients diagnosed with different joint infections, such as periprosthetic joint infection (PJI) and infectious arthritis, as well as arthrosis, reactive arthritis, and rheumatoid arthritis. The concentration of HNP1-3 was determined by HPLC. They set the cut-off concentration of SF HNP1-3 at $62.5 \mathrm{mg} / \mathrm{L}$ when comparing the results of patients with infectious arthritis versus aseptic patients with osteoarthritis. They also compared the concentration of HNP1-3 in infectious arthritis with 

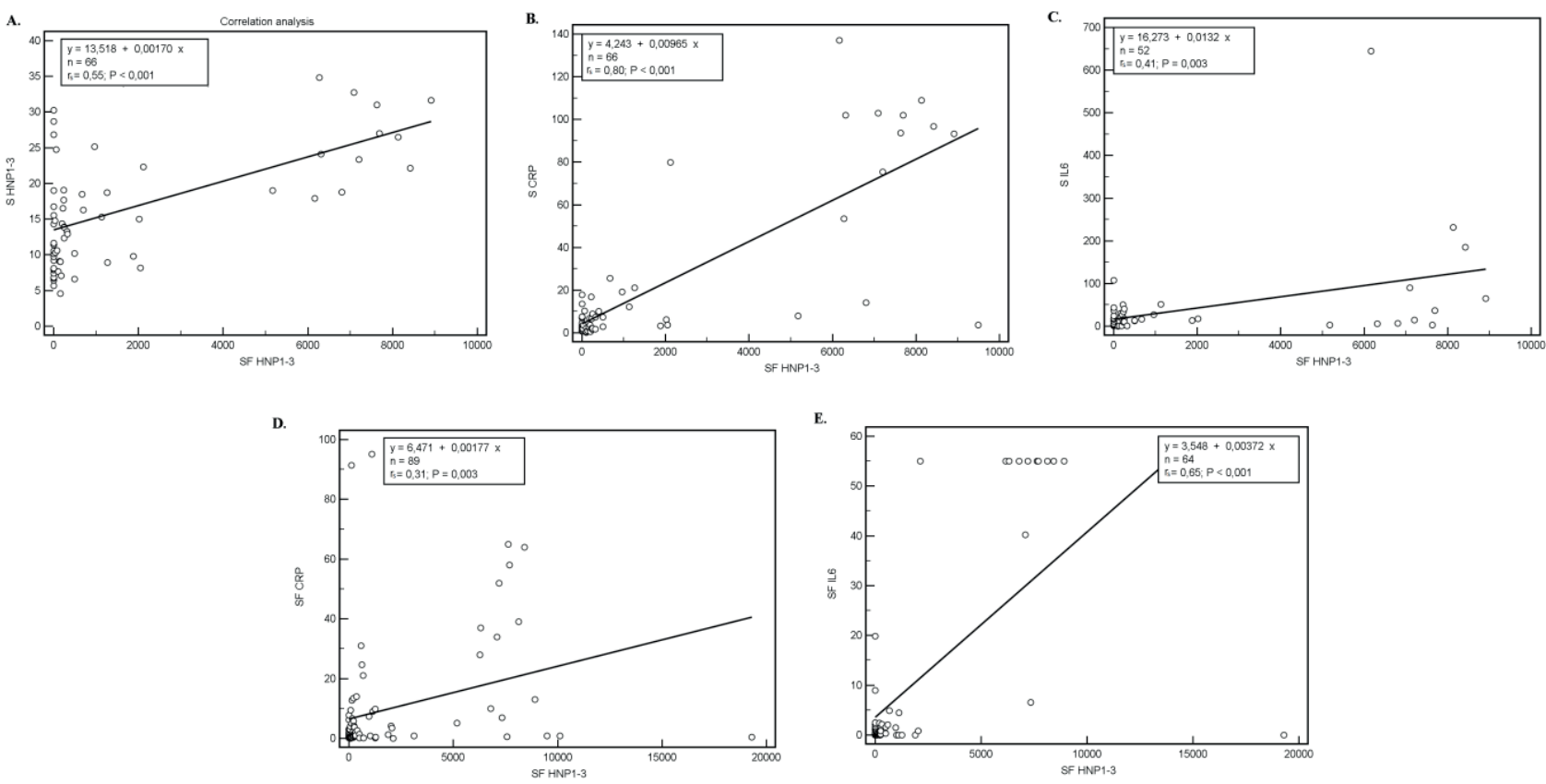

Fig. 3. Passing-Bablok regression analysis of SF HNP1-3 and S HNP1-3 (A), SF HNP1-3 and S CRP (B), SF HNP1-3 and S IL-6 (C), SF HNP1-3 and SF CRP (D), SF HNP1-3, respectively S IL-6 (E) concentrations in all groups. $r_{\mathrm{s}}=$ Spearman correlation coefficient.

aseptic patients. In this case, they set the limit concentration of HNP1-3 for infectious arthritis at $98 \mathrm{mg} / \mathrm{L}$. At the same time, due to the finding of higher values of HNP1-3 in some patients with non-infectious reactive arthritis and rheumatoid arthritis, they defined a "gray zone" in the range of $63-108 \mathrm{mg} / \mathrm{L}$.

We also tried to see whether it would be possible to replace the ELISA determination of SF HNP1-3 with a rapid laboratory test, as the most important factor in septic arthritis and other infectious diseases of joints is early diagnosis. For these purposes, we chose the determination of interleukin 6, procalcitonin, and the POCT (point-of-care testing) determination of presepsin. In addition, we investigated the possibility of replacing their determination in synovial fluid by determination in serum. Our results showed a statistically significant correlation only between SF HNP1-3 and the SF concentrations of CRP and IL-6; and between SF HNP1-3 and the serum concentrations of HNP1-3, CRP, PCT, and IL-6 in all groups.

Saleh et al. ${ }^{14}$ and Cipriano et al. ${ }^{15}$ also studied CRP, IL-6, and other biomarkers in patients with PJI, inflammatory arthritis, and non-inflammatory arthritis, and concluded that CRP remains a useful diagnostic tool with accuracy similar to that of the erythrocyte sedimentation rate (ESR), but with low specificity. Therefore, they supplemented the study with measurement of IL6 , an inflammatory cytokine produced by stimulated monocytes and macrophages. It induces the production of acute-phase proteins, including CRP, and acts as a differentiation factor for B-lymphocytes and an activating factor for T-lymphocytes. In 2010, Berbari et al. ${ }^{16}$ published a meta-analysis evaluating serum IL-6 as a potentially better diagnostic test than conventional ESR and CRP. However, recent studies ${ }^{17-20}$ have demonstrated compa- rable diagnostic performance with CRP with a sensitivity of $48 \%$, a specificity of $95 \%$, and an AUC (Area Under Curve) of 0.687 , with sensitivity and specificity ranging from $49 \%$ to $79 \%$ and $58 \%$ to $88 \%$, respectively, depending on the cut-off value used. This variability and inconsistency of results therefore limits its use as a screening test. Another parameter in our study, used as a biomarker of sepsis, was procalcitonin (PCT), a protein produced by neuroendocrine cells and parafollicular thyroid cells under physiological conditions ${ }^{21}$. Unfortunately, in the case of PJI detection, similarly to IL-6, only low sensitivity of the test was demonstrated (approximately 53\%) ( ref. $^{22}$ ), so even this parameter is not an ideal biomarker of joint infections.

The last of the studied biomarkers was presepsin, which is a tool for diagnosing severe conditions caused not only by $\mathrm{G}-$ and $\mathrm{G}+$ bacteria, but also by fungi. Presepsin is a product of the direct reaction of CD 14 protein to lipopolysaccharides, while the increase in CRP and PCT levels is indirectly influenced mostly by IL-6 $\left(\right.$ ref. $\left.^{23-27}\right)$. Therefore, elevated presepsin levels are considered a useful tool that specifically reflects the presence of infection, as opposed to CRP, which may be elevated for non-infectious reasons, such as after trauma. It is suitable for monitoring and prognosis of the patient's condition during antibiotic treatment, neonatal sepsis, for the prediction of postoperative mortality. Its activation time is around $2 \mathrm{~h}$, it reaches its maximum value in $3 \mathrm{~h}$, and its half-life is 4-5 h. Presepsin is not affected by ongoing trauma and is also considered an indicator of negative prognosis, especially in patients with liver disease. The highest sensitivity (87.8\%) and specificity (81.4\%) have been demonstrated for the cut-off plasma level of $600 \mathrm{ng} / \mathrm{L}$. Imagama et al. ${ }^{28}$ studied presepsin in synovial fluid, and presepsin and procalcitonin in the blood of 18 
patients with septic arthritis (SA), including PJI, and in 28 patients with osteoarthritis. They showed statistically significantly increased levels of presepsin in synovial fluid and blood, and of PCT in the blood in the SA group. They concluded that presepsin has the potential to be a new biomarker of septic arthritis. At the same time, Marazzi et al. ${ }^{29}$ examined this marker in patients with PJI and demonstrated its higher diagnostic value compared with CRP and IL-6. However, in our study, when comparing presepsin with HNP1-3, no statistically significant correlation was demonstrated in either serum or synovial fluid. Yet, it cannot be said that presepsin is not a suitable biomarker of sepsis in joint effusions. The above authors only compared presepsin, or other biomarkers (CRP, IL6 , and others) in the group of patients with PJI versus osteoarthritis, but not compared to HNP1-3.

In consequence, in addition to HNP1-3, IL-6 and CRP both appeared to be a suitable biomarker that enables distinguishing between infectious and non-infectious agents of joint disease in both synovial fluid and serum. Furthermore, due to their 24-hour availability in the laboratory of clinical biochemistry, they are preferred biomarkers over HNP1-3, which can be determined only by using ELISA or the Synovasure Alpha Defensin Test.

To evaluate diagnostic validity we used Cohen's kappa statistic. The highest kappa coefficient was demonstrated between the concentrations of SF HNP1-3 and SF IL-6. This supports the previous conclusions that we could routinely use both tested biomarkers to differentiate between infectious and non-infectious causes of joint diseases.

\section{Limits of the study}

Our study has several weaknesses. Even though 3-5 $\mathrm{SF}$ and serum aliquots were frozen for most patients in the study, there was not always enough material, especially serum, for all the tests. Some samples of synovial fluid were so viscous that they could not be analyzed. The patient samples were obtained during a 2-year study $(n=101)$, but no patient had confirmed septic arthritis. Finally, detailed clinical characteristics of patients within individual groups were not taken into account in the analysis; instead, we followed the laboratory results, according to which the samples were divided into individual groups.

\section{CONCLUSIONS}

In this study, we tested a diagnostic kit for the determination of HNP1-3 concentrations in biological fluids. The HNP1-3 ELISA assay has adequate sensitivity and is suitable for SF and $\mathrm{S}$ analysis. We estimated the reference limits for SF and S HNP1-3. The data showed good correlation and moderate conformity between HNP1-3 and IL-6 synovial fluid concentrations. When assessing the relationship of HNP1-3 concentrations and type of synovial fluid, correlations were found between the concentrations of SF HNP1-3 and SF CRP and SF IL-6; and between SF HNP1-3 and S HNP1-3, S CRP, and S IL-6 in all groups. The results support the hypothesis that SF
HNP1-3 is a suitable biomarker to use to distinguish between inflammatory and non-inflammatory joint diseases.

\section{Acknowledgments}

We would like to thank laboratory technicians Lenka Furstova, Ilona Faruzelova, Renata Maleckova, Renata Vytiskova and Blanca Strakosova for their expert technical assistance.

The study was supported by the Ministry of Health, the Czech Republic - conceptual development of research organization (FNOs/2018). The funder had no role in designing the study, data collection and analysis, the decision to publish, or preparation of the manuscript. There was no additional external funding received for this study.

Author contributions: PK, IB, PW, RH: designed the study; PK, IB: performed and evaluated biochemical markers; PK, IB, PW, RH: clinical data collection and management; PK, IB, PW, RH, FV: analyzed the data; PK, IB, PW, RH: wrote the first draft of the manuscript; PK, IB, PW, RH, FV, DS: editing of the manuscript; PK, IB, PW, RH, FV, DS: read and approved the final version of the manuscript.

Conflict of interest statement: The authors state that there are no conflicts of interest regarding the publication of this article.

\section{REFERENCES}

1. Dungl P. Orthopedics, Grada Publishing, a.s. 2014; 23-25. ISBN 97880-247-4357-8.

2. Selsted ME, Harwig SS, Ganz T, Schilling JW, Lehrer RI. Primary structures of three human neutrophil defensins. J Clin Invest 1985;76:1436-39.

3. Ganz T, Selsted ME, Szklarek D, Harwig SS, Daher K, Bainton DF, Lehrer RI. Defensins. Natural peptide antibiotics of human neutrophils. J Clin Invest 1985;76:1427-35.

4. Zhang Y, Lu W, Hong M. The membrane-bound structure and topology of a human a-defensin indicate a dimer pore mechanism for membrane disruption. Biochemistry 2010;49(45):9770-82.

5. Human HNP1-3 ELISA Kit, Product information and manual, HycultBiotech, Edition 08-16. [online]. In: https://www.hycultbiotech.com/hk317-01 [cit. 2020-10-06].].

6. McHugh ML. Interrater reliability: the kappa statistic. Biochemia Medica 2012;22(3):276-82.

7. Stata Version 13, Stata Press Publication, Texas, 2013. ISBN-13:9781-59718-129-7.

8. Clinical and Laboratory Standards Institute (CLSI). Defining, establishing, and verifying reference intervals in the clinical laboratory; approved guideline. CLSI document C28 - A3. 3rd ed. Wayne: PA: Clinical and Laboratory Standards Institute; 2008.

9. CRP Latex, Beckman Coulter, Inc. 250S. Kraemer Blvd., Brea, CA 92821 USA. Version: 2013-07.

10. Interleukin 6 (IL6), Siemens Healthare Diagnostics Inc. Version: RPBL1223/R4_CS Rev. D, 2019-10.

11. Kemik O, Kemik AS, Sumer A, Begenik H, Purisa S, Tuzun S. Human neutrophil peptides 1, 2 and 3 (HNP 1-3): elevated serum levels in colorectal cancer and novel marker of lymphatic and hepatic metastasis. Hum Exp Toxicol 2013;32(2):167-71.

12. Qi YY, Zhou XJ, Cheng FJ, Zhang H. Elevated plasma a-Defensins (HNP1-3) levels correlated with IgA1 glycosylation and susceptibility to IgA nephropathy. Dis Markers 2016;2016:8123138.

13. Melicherčík $P$, Klapková E, Kotaška K, Jahoda D, Landor I, Čeřovský V. High-performance liquid chromatography as a novel method for the determination of a-Defensins in synovial fluid for diagnosis of orthopedic infections. Diagnostics (Basel) 2020;10(1):33. 
14. Saleh A, George J, Faour M, Klika AK, Higuera CA. Serum biomarkers in periprosthetic joint infections. Bone Joint Re. 2018;7(1):85-93.

15. Cipriano CA, Brown NM, Michael AM, Moric M, Sporer SM, Della Valle CJ. Serum and synovial fluid analysis for diagnosing chronic periprosthetic infection in patients with inflammatory arthritis. J Bone Joint Surg [Am] 2012;94-A:594-600.

16. Berbari E, Mabry T, Tsaras G, Spangehl M, Erwin PJ, Murad MH, Steckelberg J, Osmon D. Inflammatory blood laboratory levels as markers of prosthetic joint infection: a systematic review and metaanalysis. J Bone Joint Surg [Am] 2010;92-A:2102-9.

17. Glehr M, Friesenbichler J, Hofmann G, Bernhardt GA, Zacherl M, Avian A, Windhager R, Leithner A. Novel biomarkers to detect infection in revision hip and knee arthroplasties. Clin Orthop Relat Res 2013;471(8):2621-28.

18. Ettinger M, Calliess T, Kielstein JT, Sibai J, Brückner T, Lichtinghagen $\mathrm{R}$, Windhagen $\mathrm{H}$, Lukasz A. Circulating biomarkers for discrimination between aseptic joint failure, low-grade infection, and high-grade septic failure. Clin Infect Dis 2015;61:332-41.

19. Gollwitzer $H$, Dombrowski $Y$, Prodinger PM, Peric $M$, Summer $B$ Hapfelmeier A, Saldamli B, Pankow F, von Eisenhart-Rothe R, Imhoff $A B$, Schauber J, Thomas P, Burgkart R, Banke IJ. Antimicrobial peptides and proinflammatory cytokines in periprosthetic joint infection. J Bone Joint Surg Am 2013;95(7):644-51.

20. Randau TM, Friedrich MJ, Wimmer MD, Reichert B, Kuberra D, StoffelWagner B, Limmer A, Wirtz DC, Gravius S. Interleukin-6 in serum and in synovial fluid enhances the differentiation between periprosthetic joint infection and aseptic loosening. PLoS One 2014;9:e89045.

21. Prucha M, Bellingan G, Zazula R. Sepsis biomarkers. Clin Chim Acta 2015;440:97-103.

22. Xie K, Qu X, Yan M. Procalcitonin and a-defensin for diagnosis of periprosthetic joint infections. J Arthroplasty 2017;32:1387-94.
23. Agilli M, Sener I, Yesildal F, Honca T, Aydin I, Akgul EO, Yaman H. A new marker for the diagnosıs of sepsis: Presepsin. J Investig Biochem 2012;1(1):55-7.

24. Faix JD. Presepsin - the new kid on the sepsis block. Clin Biochem 2014;47(7-8):503-4.

25. Kim H, Hur M, Moon HW, Yun YM, Di Somma S; GREAT Network Multi-marker approach using procalcitonin, presepsin, galectin-3, and soluble suppression of tumorigenicity 2 for the prediction of mortality in sepsis. Ann Intensive Care 2017;7(1):27.

26. Masson S, Caironi $P$, Spanuth E, Thomae R, Panigada M, Sangiorgi G, Fumagalli R, Mauri T, Isgrò S, Fanizza C, Romero M, Tognoni G, Latini R, Gattinoni L; ALBIOS Study Investigators. Presepsin (soluble CD14 subtype) and procalcitonin levels for mortality prediction in sepsis: data from the Albumin Italian Outcome Sepsis trial. Crit Care 2014;18(1):R6.

27. Sparacino M, Russo E, Vitali S, Torri A, Pedna M F, Di Viesti M, Gagliardi M, Crepaldi E, Castiglioni M, Agnoletti V, Sambri V. Plasma concentration of presepsin and its relationship to the diagnosis of infections in multiple trauma patients admitted to intensive care. Microbiologia Medica 2017;32(2).

28. Imagama T, Tokushige A, Seki K, Seki T, Nakashima D, Ogasa H, Sakai T, Taguchi T. Early diagnosis of septic arthritis using synovial fluid presepsin: A preliminary study. J Infect Chemother 2019;25(3):170-4

29. Marazzi MG, Randelli F, Brioschi M, Drago L, Romanò $C L$, Banfi G, Massaccesi L, Crapanzano C, Morelli F, Corsi Romanelli MM, Galliera E. Presepsin: A potential biomarker of PJI? A comparative analysis with known and new infection biomarkers. Int J Immunopathol Pharmacol 2018;31:394632017749356. 